\title{
Structure of mixed acid/decyl monolayers grafted on oxide free Si(111) surfaces
}

\author{
Catherine Henry-de-Villeneuve*, Thang Long Nguyen-Le, François Ozanam, \\ Philippe Allongue* \\ Laboratoire de Physique de la Matière Condensée, Ecole Polytechnique, UMR7643 CNRS, \\ Route de Saclay, F-91128 Palaiseau France.
}

\begin{abstract}
The structure of mixed acid / decyl monolayers (MLs) grafted on oxide free Si(111) surfaces by photochemical hydrosilylation in a mixture of neat undecylenic acid and 1-decene is studied in detail. After appropriate surface cleaning of as grafted surfaces, AFM (topography and phase imaging) and calibrated FTIR analysis demonstrate that a mixed monolayer is formed, free of residue. When the acid-molecule fraction $\left(\Gamma_{\mathrm{SOL}}\right)$ is $>0.1$ mixed monolayers are homogeneous on the scale of observations and they are only slightly enriched in acid chains with respect to the solution. Conversely, when $\Gamma_{\mathrm{SOL}}<0.1$ the acid chain fraction within the ML becomes quasiindependent of the solution composition and may become much larger than $\Gamma_{\mathrm{SOL}}$. In addition, dark domains are observed in AFM phase images. Correlations between the characteristic parameters of vCO IR band and AFM phase images suggest a strong phase separation of acid and alkyl chains at various length scales. A model involving a structuration of the grafting solution is proposed to explain observations.
\end{abstract}

Keywords: surface functionalization, mixed monolayer, carboxylic acid monolayer, silicon, IR spectroscopy, AFM

* Corresponding authors: Catherine Henry de Villeneuve (e-mail: catherine.henry-devilleneuve@ polytechnique.edu); Philippe Allongue (e-mail: philippe.allongue @ polytechnique.edu); 


\section{Introduction}

Self-assembled monolayers (SAMs) consisting of more than one molecular component (mixed monolayers) are important for chemical separation, ${ }^{1}$ Langmuir-Blodgett ${ }^{2}$ and in surface science ${ }^{3}$, 4,5,6,7,8 because mixed monolayers are frequently used to tailor surface properties such as wetting ${ }^{9,10,11}$ and work function. ${ }^{12,11}$ Bio-sensing systems ${ }^{13,14}$ make also use of mixed-monolayers that contain specific sites for anchoring the biomolecules in a controlled amount and complementary sites to prevent non-specific adsorption of the target molecule. However, the spatial arrangement of the components within the monolayer is very often overlooked in the literature although this is a very important issue to obtain the targeted functionality. In other words, it is important to study the monolayer structure to know whether the different components are phase separating or form a solid solution.

There are different approaches to prepare a mixed monolayer. The most popular and simplest one consists in co-adsorbing (or co-reacting) the different components on a surface. The formation of such monolayer is a dynamic process where the adsorption energy, the interactions between adsorbed molecules and the interactions between molecules in the monolayer and in solution, play a key role. In the case of a multifunctional monolayer, pair interaction energies must be considered and these will depend, for instance, on relative length and/or structure of the chain and also on their terminal groups. Two categories of systems may therefore be considered. (i) Systems for which the adsorption energy is low enough to allow a reorganization of the molecules to take place via desorption from the surface and/or a surface mobility. In this case, the monolayer formation process is close to thermodynamic equilibrium and phase segregation may be expected when the contribution of the interaction between molecules overcomes that of entropic origin. (ii) Systems for which the surface linkage is such strong that it becomes 
irreversible in the conditions of the functionalization. In this case a simple statistical repartition of the grafted chains is rather anticipated and therefore perfect mixing should occur.

The functionalization of gold with thiol derivatives ${ }^{3,4}$ and oxides with silane, ${ }^{4,6,7}$ which is widely used in various contexts, obviously belongs to the first category of systems. A maximum packing is achieved after several hours or days of incubation and phase segregation is generally observed in mixed monolayer on $\mathrm{Au}{ }^{9,15,16,17,18,19,20}$ or on oxides ${ }^{4,6,7}$ depending on the relative length of the different chains and their respective terminal groups. Conversely, the functionalization of oxide-free Si surfaces ${ }^{4,14}$ belongs to the second category of systems because the formed $\mathrm{Si}-\mathrm{C}$ bond is such strong (binding energy $\sim 4.5 \mathrm{eV}$ ) and chemically stable that it cannot be cleaved at temperatures below $150^{\circ} \mathrm{C}^{21,22}$ Mixed monolayers have been prepared on silicon ${ }^{23,24,25}$ but their composition is generally assessed qualitatively only and is more rarely quantified. ${ }^{23}$ To date, there are no studies describing the structure of mixed monolayers on oxidefree silicon. Titration experiments indirectly suggest that carboxy-alkyl and alkyl chains phase separate on the surface. ${ }^{26}$

In this work, we investigate the composition and structure of mixed monolayer prepared by photochemical reaction of atomically flat $\mathrm{H}$-terminated $\mathrm{Si}(111)$ surfaces with a solution composed of 1-decene and undecylenic acid in variable proprotions. We present AFM observations and calibrated IR spectroscopy characterizations over a wide range of acid chain concentrations, exploring in particular extreme dilution of acid chains in decene. At low dilution (acid molecule fraction $\Gamma_{\mathrm{SOL}}>0.1$ in the grafting solution), results confirm those of Faucheux et al., ${ }^{23}$ but new features are observed as soon as $\Gamma_{\mathrm{SOL}}$ becomes smaller than 0.1. Quantitative correlations between AFM phase images and IR spectroscopic data are used to rationalize the 
observations and discuss the structure of the mixed monolayers as function of the composition of the grafting solution. A schematic mechanism is proposed.

\section{Experimental}

Preparation of the H-Si(111) surfaces: Silicon samples were cut in n-type $\mathrm{Si}(111)$ wafers with $0.2^{\circ}$ miscut angle along the [11-2] direction (Siltronix, France). One-side polished (CZ purified, 1-10 $\Omega . \mathrm{cm}$ ) and double-side polished wafers (FZ-purified, 10-60 $\Omega . \mathrm{cm}$ ) were used for AFM characterizations and FTIR analysis, respectively. Samples were carefully cleaned in $\mathrm{H}_{2} \mathrm{O}_{2} / \mathrm{H}_{2} \mathrm{SO}_{4}$ piranha solution ([1:2] in vol.) to remove organic contaminants and then etched in oxygen-free $40 \% \mathrm{NH}_{4} \mathrm{~F}$ solutions to obtain $\mathrm{H}$-terminated surface with a staircase structure at the

atomic scale. ${ }^{27}$ After etching the samples were rinsed with ultrapure water (UPW 18.2 M 2 , Millipore ${ }^{\circledR}$ ) and carefully dried and stored under argon until their introduction into the grafting solution.

Surface functionalization: Organic monolayers (MLs) were prepared by photochemical hydrosilylation (UV light $312 \mathrm{~nm}, 6 \mathrm{~mW} / \mathrm{cm}^{2}$ ) in a mixture of neat undecylenic acid (99\% Acros Organics) and neat decene (97\% Sigma-Aldrich). As previously reported, ${ }^{23}$ a freshly prepared H$\operatorname{Si}(111)$ surface was introduced into the grafting solution outgassed by argon bubbling. The reaction time was $3 \mathrm{~h}$ to $4 \mathrm{~h}$.

Post-cleaning procedure: In order to obtain atomically smooth functionalized surface free of residual reaction products and/or solvent, different rinsing procedures were used. In the case of mixed monolayers prepared in solutions with acid chain fraction greater than 0.1 or decyl monolayers, glacial hot acetic acid (RSE Carlo Erba) or THF and dichloromethane (DCM) HPLC grade were used, respectively, as previously described. ${ }^{23,28}$ In the case of mixed 
monolayers prepared in solutions with acid chain fraction smaller than 0.1 , a mechanical cleaning was applied as shown in Supplementary Information (Fig. S1-S2). After a final rinse in hot acetic acid $\left(70^{\circ} \mathrm{C}\right.$ for $\left.20 \mathrm{~min}\right)$ and boiling dichloromethane $(10 \mathrm{~min})$, atomically smooth surfaces, exempt of residues, were routinely obtained.

AFM observations: AFM characterizations were performed under $\mathrm{N}_{2}$ atmosphere using a PicoSPM (Molecular Imaging) AFM operating in AC Mode. Sharp silicon tips (nominal tip radius $\leq 7 \mathrm{~nm}$ ) were used for high-resolution imaging (PointProbesPlus, Nanosensors, spring constant $\sim 40 \mathrm{~N} \mathrm{~m}^{-1}$, resonance frequency $\sim 160 \mathrm{kHz}$ ). Imaging was carried out in the attractive force regime, where the tip is in very weak interaction with the surface so as to achieve high resolution and non-destructive imaging of soft materials. ${ }^{29,30}$ Topography and phase images were recorded simultaneously to investigate the topography and the chemical homogeneity of the surfaces, respectively. ${ }^{29}$

FTIR measurements: FTIR measurements were performed in ATR geometry with an Equinox 55 spectrometer (Bruker, Germany) equipped with a liquid-nitrogen cooled Mercury-Cadmium Telluride photovoltaic detector. The ATR samples (FZ-purified Si(111) wafer, thickness 500-550 $\mu \mathrm{m})$ were shaped as prisms with two opposite sides bevelled at $\sim 45^{\circ}$. Their length $(\sim 15 \mathrm{~mm}$, for ca $\sim 30$ internal reflections) was chosen to obtain wide-range FTIR spectra $\left(1000-4000 \mathrm{~cm}^{-1}\right)$. Measurements were carried out in a $\mathrm{N}_{2}$-purged chamber to minimize $\mathrm{H}_{2} \mathrm{O}$ vapour and $\mathrm{CO}_{2}$ absorption. True quantitative determination of the composition of the monolayers was performed using a specially developed FTIR analysis method based on absorbance measurements in ATR geometry using polarized IR beam and a calibration of the absorbance cross sections of specific IR vibrations, using the same geometry. ${ }^{23}$ 


\section{Results:}

Figures 1-2 display AFM topography and phase images of samples grafted in solutions with variable acid chain fractions $\left(\Gamma_{\mathrm{SOL}}\right)$ whose value is noted above each image. All images (except that of Fig. 1b) have been acquired on samples subjected to an appropriate cleaning procedure after grafting. Globally all surfaces are smooth with a staircase structure similar to that of the etched $\mathrm{H}-\mathrm{Si}(111)$ surface with flat terraces separated by $3.1 \AA$ high steps corresponding to two $\mathrm{Si}(111)$ atomic planes. For $\Gamma_{\mathrm{SOL}}>0.1$ (Fig. 1a) a standard chemical rinsing in THF followed by hot acetic acid ${ }^{23,28}$ is sufficient to obtain such residue free surface. At extreme dilution $\left(\Gamma_{\mathrm{SOL}}\right.$ $=10^{-4}$, Fig. 1b), the same rinsing procedure leaves a quasi-perfect surface with however a homogeneous repartition of protrusions exhibiting narrow size distribution (height $\sim 1 \mathrm{~nm}$, see cross section in Fig. 1b1). Applying a mechanical cleaning leaves an atomically smooth surface (see Fig. 1c). At intermediate $\Gamma_{\text {SOL }}$ values $\left(10^{-3}<\Gamma_{\text {SOL }}<0.1\right)$ AFM and optical images reveal that a large amount of matter is left on as grafted surfaces, in spite of repeated rinsing in solvents (see supplementary information, Figs. S1-S2). A mechanical cleaning is indispensable to obtain residue-free surfaces as in Fig. 2.

Figures 1a1, c1 and 2a1-b1 show phase images recorded simultaneously with the topography images shown above. At low dilution (image 1a1) the phase is constant which suggests a homogeneous monolayer on the $\mathrm{nm}$ scale. At extreme dilution (image 1c1 and inset), dark nanoscale domains (diameter $\sim 8 \mathrm{~nm}$ ) are easily observable due to a clear white / black contrast. The domain coverage is $\sim 0.08( \pm 0.03)$ and image analysis gives a domain density $\left(\sim 1.610^{11}\right.$ domain $\left./ \mathrm{cm}^{2}\right)$ that is close to the density of protrusions seen in Fig. $1 \mathbf{b}\left(\sim 10^{11} / \mathrm{cm}^{2}\right)$. Therefore, phase domains are likely related to the protrusions observed prior to full surface cleaning. At intermediate dilution, wide circular dark domains of diameter $0.2-2 \mu \mathrm{m}$ are visible 
(images $2 \mathrm{a} 1,2 \mathrm{~b} 1$ ), which will be called $\mu$-domains in the following. Outside $\mu$-domains (inset of Fig. 2b1), a real phase contrast on the nm scale is found again, which we assign to nanoscale domains of a few tens of $\mathrm{nm}$. These domains are called n-domains in the following. The coverage of n-domains falls in the range $0.2-0.5$ and giving a more precise value turned out to be uncertain because of the difficulty to set properly an image background plane.

Figure 3 presents a plot of the $\mu$-domain coverage as a function of $\Gamma_{\mathrm{SOL}}$. The grey shaded area outlines the restricted range of acid molecule fraction where $\mu$-domains are observed. This coverage does not account for the coverage of n-domains. The red data point for $\Gamma_{\mathrm{SOL}}=10^{-4}$ corresponds to the dark domains resolved in Fig. 1c1.

Wide-range FTIR characterization confirms that the surfaces are covered by an organic monolayer. The silicon substrate is only weakly oxidized, with an average thickness of silicon oxide smaller than $0.15 \mathrm{~nm}$ (essentially formed during the cleaning procedure, see supplementary information Fig. S5). Fig.4a and 4b display narrow-range FTIR spectra of grafted monolayers after cleaning. The band centered at $\sim 1715 \mathrm{~cm}^{-1}$ (Fig. 4a) is assigned to the $v \mathrm{C}=\mathrm{O}$ vibration mode of carboxylic groups and the different bands in Fig. $\mathbf{4 b}$ are assigned to the $v \mathrm{CH}_{3}(\sim 2960$ and $\left.\sim 2880 \mathrm{~cm}^{-1}\right)$ and $\mathrm{vCH}_{2}\left(\sim 2921\right.$ and $\left.2851 \mathrm{~cm}^{-1}\right)$ vibration modes of alkyl chains (decyl and carboxydecyl). The presence of these IR bands is consistent with the grafting of an organic film composed of acid- and methyl-terminated alkyl chains. Figure $4 \mathbf{c}$ shows a plot of the total surface density of grafted chains (open circle) and of the number of acid chains (bold squares) as derived from the calibration of the $\mathrm{vCH}$ and $v \mathrm{CO}$ bands. ${ }^{23}$ Quite satisfying is that the total surface density $N=N_{\text {decyl }}+N_{\text {acid }}$ stays around $\sim 2.5$ chains $/ \mathrm{nm}^{2}$ for all the $\Gamma_{\text {SOL }}$ values. This density corresponds to 1 chain per $\sim 3 \mathrm{Si}$ surface atoms (there are $7.8 \mathrm{Si}$ atoms $/ \mathrm{nm}^{2}$ on a perfect 
H-terminated $\mathrm{Si}(111)$ surface). There is a slight decrease of the total chain density with increasing $\Gamma_{\text {SOL }}$ which we attribute to the fact that $-\mathrm{COOH}$ terminal groups are bulkier than $-\mathrm{CH}_{3}$ groups. A straight way of comparing the monolayer composition with that of the solution is plotting the acid chain fraction in the monolayer $\left(\Gamma_{\mathrm{SURF}}\right)$ as a function of $\Gamma_{\mathrm{SOL}}(\mathbf{F i g}$. $4 \mathbf{c}$, open squares, right y-axis). From this graph, one immediately captures that $\Gamma_{\text {SURF }}$ stays quasi-constant in the range $0.3-0.4$ over a wide range of grafting solution composition, corresponding to a surface density of $0.7-1$ acid chains $/ \mathrm{nm}^{2}$. An extreme dilution $\left(\Gamma_{\mathrm{SOL}} \leq 10^{-4}\right)$ must be used to decrease significantly $\Gamma_{\mathrm{SURF}}$. For $\Gamma_{\mathrm{SOL}} \geq 0.2, \Gamma_{\mathrm{SURF}} \sim \Gamma_{\mathrm{SOL}}$ in quantitative agreement with published data. $^{23}$

The strange behavior observed for $\Gamma_{\mathrm{SOL}} \leq 0.1$ motivated specific experiments where a $\mathrm{H}$ terminated surface is exposed to mixed solutions in the dark, i.e., in conditions such that no grafting occurs. Fig. 5 plots the surface density of acid chains physisorbed on the $\mathrm{H}-\mathrm{Si}(111)$ surface as a function of $\Gamma_{\mathrm{SOL}}$. The data are derived from in-situ FTIR spectra given in supplementary information (Fig. S6). The log-log scale helps for visualizing that the adsorption of acid chains starts from an acid fraction as low as $\Gamma_{\mathrm{SOL}}=10^{-4}$ (this dilution corresponds to an acid chain concentration of $\sim 0.5 \mathrm{mM})$. Saturation of the surface is reached for $\Gamma_{\mathrm{SOL}} \sim 10^{-2}(50$ $\mathrm{mM}$ ) and there are about 10 acid chains $/ \mathrm{nm}^{2}$ in this case. Corresponding AFM images of $\mathrm{H}$ terminated surfaces exposed to grafting solutions in the dark are presented in Fig. 6. The surface exposed to pure decene (Fig. 6a) presents nm-white spots of height $0.4 \mathrm{~nm}$ assigned to $\mathrm{nm}$ oxide clusters. ${ }^{31}$ For increasing values of $\Gamma_{\text {SOL }}$ (Figs. 6b-d), the surface is progressively covered with spots and saturation occurs for $\Gamma_{\mathrm{SOL}}=0.01$ in agreement with Fig. 5. The spots height is typically $1.5 \mathrm{~nm}$ in images $5 \mathrm{~b}-5 \mathrm{~d}$. Performing an accurate particle analysis proved to be very difficult due to strong tip to sample interactions. 


\section{Discussion:}

The above observations demonstrate that clean and dense mixed monolayers with variable acid chain fraction $\left(\Gamma_{\mathrm{SURF}}\right)$ may be obtained on $\mathrm{Si}(111)$ surfaces after application of an appropriate cleaning procedure.

When $\Gamma_{\mathrm{SOL}}>0.1$, the monolayer is homogenous on the scale of observations since phase images are featureless. $\Gamma_{\text {SURF }}$ is only somewhat larger than $\Gamma_{\text {SOL }}(\mathbf{F i g} .4 \mathbf{c})$. Besides, FTIR results indicate that the absorption frequency of $\mathrm{COOH}$ groups $\mathrm{f}_{\mathrm{CO}}\left(\sim 1714.8 \mathrm{~cm}^{-1}\right)$ is quasi-independent of the monolayer composition in this range of dilution while the width (fwhm) of $v \mathrm{CO}$ band progressively increases from 20 to $40 \mathrm{~cm}^{-1}$ when $\Gamma_{\text {SOL }}$ approaches 0.1 (Fig. 7a). Therefore, the acid groups remain in rather strong mutual interaction while disorder increases upon monolayer dilution by addition of non-functionalized chains. The existence of a close proximity between neighboring acid chains for all the corresponding $\Gamma_{\text {SURF }}$ values is consistent with the observation that the titration behavior of such mixed monolayers is almost independent of the monolayer composition. $^{26}$

The rest of the discussion aims at explaining the mechanism responsible for the phaseseparation suggested by AFM phase images when $\Gamma_{\mathrm{SOL}}<0.1$. In fact, a dark contrast in AFM phase images indicates an enhanced AFM tip-to-surface interaction which is plausibly assigned to a region locally enriched in hydrophilic $\mathrm{COOH}$ groups. Complementary information about the molecular environment of $\mathrm{COOH}$ groups is provided by $v \mathrm{CO}$ IR band frequency $\left(\mathrm{f}_{\mathrm{CO}}\right)$, which is a sensitive probe of the interactions between $\mathrm{COOH}$ groups (essentially H-bonding), ${ }^{32}$ and its width (fwhm), which accounts for the homogeneity of the local vibrator configurations. To fix 
ideas, we recall that it is commonly accepted that $\mathrm{f}_{\mathrm{CO}} \sim 1740 \mathrm{~cm}^{-1}$ corresponds to isolated $\mathrm{COOH}$ groups while $\mathrm{f}_{\mathrm{CO}} \sim 1700-1710 \mathrm{~cm}^{-1}$ corresponds to cyclic ("face to face") acid dimers. ${ }^{32}$

In the following, the number of acid chains located in dark domains and averaged per unit surface area over the whole surface will be designated $N_{\mu, \mathrm{n}}$, with the subscripts referring to $\mu$ and n-domains, respectively. Straightforwardly: $N_{\mu, \mathrm{n}}=\theta_{\mu, \mathrm{n}} D_{\mu, \mathrm{n}}$, where $\theta_{\mu, \mathrm{n}}$ is the domain coverage and $D_{\mu, \mathrm{n}}$ is the local acid chain density in the dark $\mu$ - and n-domains, respectively.

\subsection{Structure and formation mechanism of monolayers at extreme dilution $\left(\Gamma_{\mathrm{SOL}}=10^{-4}\right)$ :}

In such conditions, a clear black/white contrast in AFM phase images strongly suggests a full phase segregation of acid chains in nanoscale domains (Fig. 1c1). Given the average density of acid chains $N_{\text {acid }}=0.23 / \mathrm{nm}^{2}$ (Fig. 4) and the coverage of n-domains $\theta_{\mathrm{n}}=0.08$, a local acid chain density $D_{\mathrm{n}}=N_{\text {acid }} / \theta_{\mathrm{n}}=2.9 / \mathrm{nm}^{2}$ is determined. The characteristic frequency of the $\mathrm{vCO}$ band $\left(\sim 1712 \mathrm{~cm}^{-1}\right)$ is nonetheless somewhat surprising (Fig. 7a) because the necessary structural chain distortions to form symmetric face-to-face acid dimers (expected frequency $\sim 1710 \mathrm{~cm}^{-1}$ ) are impossible to meet the estimated high $D_{n}$ value. A likely explanation is that the interactions between the $\mathrm{COOH}$ dynamic dipoles, which decay as $1 /(\text { distance })^{3}$, become significant at such high surface density. This point will be addressed in a forthcoming paper.

The origin of the above phase separation probably results from a structuration of the grafting solution. Molecular or supramolecular assembly of carboxylic acid chains in benzene ${ }^{33}$ or in $\mathrm{THF}^{34}$ at concentration at least down to the millimolar range was indeed reported and similar behavior may be anticipated for our mixed grafting solutions. Therefore, we propose that undecylenic acid molecules self-organize as reverse micelles when the acid chain concentration becomes close to the critical micellar concentration $(\mathrm{cmc})$. The $\mathrm{COOH}$ groups are indeed likely 
confined in the micelle core because they are stabilized by H-bonding (see frequency 1711.5 $1712.5 \mathrm{~cm}^{-1}$ of the $v \mathrm{CO}$ band in Fig. S6). $\Gamma_{\mathrm{SOL}}=10^{-4}$ corresponds to an acid chain concentration of $0.5 \mathrm{mM}$ which is close to reported cmc for non-ionic amphiphile molecules in non-polar solvents. $33,34,35$

The following mechanism is then proposed for the formation of a mixed monolayer in very dilute conditions. The micelle concentration being in the $\mu \mathrm{M}$ range (see estimate below of the number of chains per micelle), the reverse micelles are progressively adsorbing as isolated entities on the H-terminated surface in the dark (Figure 8a). Upon UV illumination, the $\mathrm{C}=\mathrm{C}$ ends of the acid chains, which are located at periphery of the reverse micelles, readily react with the hydrogenated Si surface. In other places, alkyl chains (the majority species in solution) are grafted simultaneously. An alkyl monolayer is therefore obtained with isolated nanoscale acid bilayer islands (Fig. 8b). The acid dimers are finally disrupted by mechanical cleaning, which leaves an atomically smooth surface with nanoscale acid-chain-rich islands (Fig. 8c).

This scenario is not only consistent with AFM observations but also with the IR spectra recorded before and after the mechanical cleaning that indicate the removal of $\sim 25-50 \%$ of acid chains (data not shown). A further argument in favor of this scenario is the close similarity between the diameter of the reverse micelles (see estimate below) and the domain size ( $8 \mathrm{~nm}$ in Fig. 1c1). According to the scheme in Fig. 8, the reverse micelles must contain about 300 acid chains because the dark domains are composed of $\sim 150$ acid chains (diameter $\sim 8 \mathrm{~nm}$ and $D_{n}=$ 2.9 acid chains $/ \mathrm{nm}^{2}$ ) and the mechanical wiping removes about the same number of acid chains. The micelle diameter is therefore given by the inner core diameter plus two times the chain length $(1.1 \mathrm{~nm})$. To estimate the inner core diameter, we consider a COOH packing of $4.5 \mathrm{~nm}^{-2}$ on the inner surface of the micelle (this is the density of a-COOH terminated SAM on $\mathrm{Au}(111)$ ). 
This gives a core diameter of $\sim 4.4 \mathrm{~nm}$. The micelle diameter is therefore $\sim 6.6 \mathrm{~nm}$, which is very close to the domain size. This diameter is also matching well with the lateral dimension of the adsorbing entities in the dark (Fig. 6). The height of the latter appears however shallower $(\sim 1.5$ $\mathrm{nm})$ than $6.6 \mathrm{~nm}$ because of a reorganization of the micelle when it interacts with the hydrophobic $\mathrm{H}-\mathrm{Si}(111)$ surface and/or because a mechanical deformation during AFM imaging.

\subsection{Monolayer structure and origin of invariable composition at intermediate dilution}

At intermediate dilution, the striking result is that the monolayer composition stays quasi invariant over a very wide range of composition of the grafting solution: $\Gamma_{\mathrm{SURF}}=0.3-0.4$ while $10^{-3} \leq \Gamma_{\mathrm{SOL}} \leq 0.1$. The phenomenon is strongly correlated with (i) the presence of a large excess of matter left on the as-grafted surface, even after multiple rinsing in hot solvents; (ii) the formation of acid chain-rich $\mu$ - and n-domains on the AFM phase images and (iii) a lower interaction between $\mathrm{COOH}$ groups as evidenced by the shift of the $v \mathrm{CO}$ IR bands, from 1714.8 $\mathrm{cm}^{-1}$ to $1717.2 \mathrm{~cm}^{-1}$, and its broadening (Fig. $\left.7 \mathbf{b}\right)$.

As mentioned above, dark domains in AFM phase images suggest strong segregation. To check this hypothesis, we will estimate the density of acid chains $D_{\mu, \mathrm{n}}$ in $\mu$ - and n-domains. Full segregation means that $N_{\text {acid }}=D_{\mu} \theta_{\mu}+D_{\mathrm{n}} \theta_{\mathrm{n}}$, where $N_{\text {acid }}$ is given in Fig. 4 and $\theta_{\mu}$ is given by Fig. 3. For the purpose of illustration, we consider the median value $\theta_{\mathrm{n}} \sim 0.35$ to determine $D_{\mu, \mathrm{n}}$. In a restricted subrange $\left(10^{-3} \leq \Gamma_{\mathrm{SOL}} \leq 5.10^{-3}\right)$ where the contribution of $\mu$-domains is negligible, $N_{\text {acid }}=D_{\mathrm{n}} \theta_{\mathrm{n}} \sim 0.65 / \mathrm{nm}^{2}$ which yields $D_{\mathrm{n}} \sim 1.8 / \mathrm{nm}^{2}$. For $\Gamma_{\mathrm{SOL}}$ between $5.10^{-3}$ and $10^{-1}$ and

keeping $D_{\mathrm{n}} \theta_{\mathrm{n}} \sim 0.65 / \mathrm{nm}^{2}$, a postulated value of $D_{\mu} \sim 1.7 / \mathrm{nm}^{2}$ insures that the calculated $N_{\text {acid }}$ value reaches the experimentally determined one for all the investigated $\Gamma_{\mathrm{SOL}}$ values (see open squares in Fig. $\mathbf{7 b}$ ). The acid-chain domains are therefore significantly less densely packed than 
a pure acid monolayer $\left(2.4 / \mathrm{nm}^{2}\right)$ or the nanoscale domain at extreme dilution $\left(2.9 / \mathrm{nm}^{2}\right)$ and this is coherent with two observations: (i) the surface roughness is enhanced inside the $\mu$-domains (see Fig. S4) and (ii) the acid groups are in weaker interaction $\left(\mathrm{f}_{\mathrm{CO}} \sim 1717 \mathrm{~cm}^{-1}\right.$, see Fig. 7a). A full segregation at $\mu \mathrm{m}$ and a few $10 \mathrm{~nm}$ length scales appears therefore to capture the major experimental results. However, one cannot totally exclude a minor segregation at a shorter length scale since a density of $0.1 / \mathrm{nm}^{2}$ outside dark domains is calculated if $D_{\mu, \mathrm{n}}$ is taken $10 \%$ smaller than the above values.

The origin of the different length scales of segregation may be explained by extrapolating the mechanism described in Fig. 8. The surface density of the reverse micelles progressively increases with increasing $\Gamma_{\mathrm{SOL}}$ and surface saturation is reached around $\Gamma_{\mathrm{SOL}}=10^{-2}$. The corresponding surface density of acid chains is $\sim 10$ acid chains $/ \mathrm{nm}^{2}$ (Fig. 5) or $\sim 0.03$ reverse micelles $/ \mathrm{nm}^{2}$ (there are 300 molecules / micelle, see above). These values suggest that some interpenetration of adsorbed micelles takes place, or that the lipophilic character of the hydrogenated surface induces a fusion of the micelles to form bigger supramolecular structures. ${ }^{34,35}$ Upon UV irradiation, the chains belonging to these structures are again grafted preferentially on the $\mathrm{H}-\mathrm{Si}$ surface in agreement with the AFM observations after partial removal of the extra material left upon grafting (supplementary information Fig. S7). The IR data in Fig. S2 provide also strong evidence that some photochemical reactions occur in parallel in the solution phase next to the surface since the IR band related to $v \mathrm{C}=\mathrm{C}$ (expected at $\sim 1640 \mathrm{~cm}^{-1}$ ) is missing in the removed product and esters are also forming (see shoulder around $1735 \mathrm{~cm}^{-1}$ in the vCO IR band in Fig. S2). Such a reticulation of chains explains well the strong difficulties to clean the as grafted surfaces in this range of dilution. It could be favored by a dense packing of acid chains in the surface vicinity. ${ }^{36}$ 


\section{Conclusion}

The composition and the structure of mixed acid/decyl monolayers covalently grafted on oxidefree silicon surfaces by hydrosylilation have been investigated. Monolayers prepared with a high concentration of acid molecules in solution are homogenous on the $\mathrm{nm}$ scale, in perfect agreement with our previous work. Upon dilution of undecylenic acid in decene, the results demonstrate that the mixed monolayers are phase separated whereas statistical mixing was anticipated from simple considerations based on the irreversibility of the grafting reaction.

Exploring extreme dilutions was a decisive step to build a scenario and demonstrate that the phase separation is induced by a structuration of the solution. The nanoscale domains result in fact from the adsorption of individual reverse micelles composed of acid chains with their $\mathrm{COOH}$ groups inside the core of the micelle. This approach might be used for monolayer templating strategies.

At intermediate dilution, full segregation occurs again but at various length scales $(\mu \mathrm{m}$ and a few $10 \mathrm{of} \mathrm{nm}$ ) according to a process governed by a complex structuration of the grafting solution, in the bulk volume and at interfaces where some reticulation occurs. This scenario explains that the acid-chain fraction on the surface stays quasi constant over a wide range of dilution and that the excess matter left on the as grafted surface is insoluble and must be mechanically removed.

\section{Supporting information:}

This material is available free of charge via the Internet at http://pubs.acs.org. It presents AFM observations of the sample at different stages of cleaning and compares the topography inside 
and outside the dark phase domains. Full range IR spectra of the samples are presented as a complement to the narrow spectra presented in the main manuscript. Raw data corresponding to adsorption experiments are also presented. 


\section{References}

(1) Wirth, M. J., Fairbank, R. W. P., and Fatunmbi, H. O., Mixed Self-Assembled Monolayers in Chemical Separations, Science 275 (5296), 44 (1997).

(2) Dynarowicz-Łạtka, P. and Kita, K., Molecular interaction in mixed monolayers at the air/water interface, Advances in Colloid and Interface Science 79 (1), 1 (1999).

(3) Love, J. C., Estroff, L. A., Kriebel, J. K., Nuzzo, R. G., and Whitesides, G. M., SelfAssembled Monolayers of Thiolates on Metals as a Form of Nanotechnology, Chem. Rev. 105 (4), 1103 (2005).

(4) Ulman, A., Formation and structure of self-assembled monolayers, Chemical Reviews 96 (4), 1533 (1996).

(5) Buriak, J. M., Organometallic Chemistry on Silicon and Germanium Surfaces, Chem. Rev. 102 (5), 1271 (2002).

(6) Sagiv, J., Organized monolayers by adsorption. 1. Formation and structure of oleophobic mixed monolayers on solid surfaces, Journal of the American Chemical Society 102 (1), 92 (1980).

(7) Silberzan, P., Leger, L., Ausserre, D., and Benattar, J. J., Silanation of silica surfaces. A new method of constructing pure or mixed monolayers, Langmuir 7 (8), 1647 (1991).

(8) Thissen, P., Seitz, O., and Chabal, Y. J., Wet chemical surface functionalization of oxidefree silicon, Progress in Surface Science 87 (9â€ “12), 272 (2012).

(9) Laibinis, P. E., Nuzzo, R. G., and Whitesides, G. M., Structure of monolayers formed by coadsorption of two n-alkanethiols of different chain lengths on gold and its relation to wetting, J. Phys. Chem. 96 (12), 5097 (1992).

(10) Laibinis, P. E., Whitesides, G. M., Allara, D. L., Tao, Y. T., Parikh, A. N., and Nuzzo, R. G., Comparison of the structures and wetting properties of self-assembled monolayers of nalkanethiols on the coinage metal surfaces, copper, silver, and gold, Journal of the American Chemical Society 113 (19), 7152 (1991).

(11) Chen, C.-Y., Wu, K.-Y., Chao, Y.-C., Zan, H.-W., Meng, H.-F., and Tao, Y.-T., Concomitant tuning of metal work function and wetting property with mixed self-assembled monolayers, Organic Electronics 12 (1), 148 (2011).

(12) Crivillers, N., Osella, S., Van Dyck, C., Lazzerini, G. M., Cornil, D., Liscio, A., Di Stasio, F., Mian, S., Fenwick, O., Reinders, F., Neuburger, M., Treossi, E., Mayor, M., Palermo, V., Cacialli, F., Cornil, J., and Samorì, P., Large Work Function Shift of Gold Induced by a Novel Perfluorinated Azobenzene-Based Self-Assembled Monolayer, Advanced Materials 25 (3), 432 (2012).

(13) Wink, T., J. van Zuilen, S., Bult, A., and P. van Bennekom, W., Self-assembled Monolayers for Biosensors, Analyst 122 (4), 43R (1997).

(14) Ciampi, S., Harper, J. B., and Gooding, J. J., Wet chemical routes to the assembly of organic monolayers on silicon surfaces via the formation of $\mathrm{Si}-\mathrm{C}$ bonds: surface preparation, passivation and functionalization, Chemical Society Reviews 39 (6), 2158 (2010).

(15) Folkers, J. P., Laibinis, P. E., Whitesides, G. M., and Deutch, J., Phase behavior of twocomponent self-assembled monolayers of alkanethiolates on gold, J. Phys. Chem. 98 (2), 563 (1994).

(16) Stranick, S. J., Parikh, A. N., Tao, Y. T., Allara, D. L., and Weiss, P. S., Phase Separation of Mixed-Composition Self-Assembled Monolayers into Nanometer Scale Molecular Domains, J. Phys. Chem. 98 (31), 7636 (1994). 
(17) Brewer, N. J. and Leggett, G. J., Chemical Force Microscopy of Mixed Self-Assembled Monolayers of Alkanethiols on Gold: Evidence for Phase Separation, Langmuir 20 (10), 4109 (2004).

(18) Li, L., Chen, S., and Jiang, S., Molecular-Scale Mixed Alkanethiol Monolayers of Different Terminal Groups on Au(111) by Low-Current Scanning Tunneling Microscopy, Langmuir 19 (8), 3266 (2003).

(19) Collman, J. P., Devaraj, N. K., Eberspacher, T. P. A., and Chidsey, C. E. D., Mixed Azide-Terminated Monolayers: A Platform for Modifying Electrode Surfaces, Langmuir 22 (6), 2457 (2006).

(20) Alévêque, O., Levillain, E., and Morille, Y., Electroactive mixed self-assembled monolayers: A numerical overview of phase segregations, Electrochemistry Communications 45, 17 (2014).

(21) Faucheux, A., Yang, F., Allongue, P., Villeneuve, C. H. d., Ozanam, F., and Chazalviel, J. N., Thermal decomposition of alkyl monolayers covalently grafted on (111) silicon, Appl. Phys. Lett. 88 (19), 193123 (2006).

(22) Faucheux, A., Gouget-Laemmel, A. C., Allongue, P., Henry de Villeneuve, C., Ozanam, F., and Chazalviel, J. N., Mechanisms of Thermal Decomposition of Organic Monolayers Grafted on (111) Silicon, Langmuir 23 (3), 1326 (2006).

(23) Faucheux, A., Gouget-Laemmel, A. C., de Villeneuve, C. H., Boukherroub, R., Ozanam, F., Allongue, P., and Chazalviel, J. N., Well-defined carboxyl-terminated alkyl monolayers grafted onto H-Si(111): Packing density from a combined AFM and quantitative IR study, Langmuir 22 (1), 153 (2006).

(24) Lattimer, J. R. C., Brunschwig, B. S., Lewis, N. S., and Gray, H. B., Redox Properties of Mixed Methyl/Vinylferrocenyl Monolayers on Si(111) Surfaces, The Journal of Physical Chemistry C 117 (51), 27012 (2013).

(25) Fabre, B. and Hauquier, F., Single-Component and Mixed Ferrocene-Terminated Alkyl Monolayers Covalently Bound to Si(111) Surfaces, The Journal of Physical Chemistry B 110 (13), 6848 (2006).

(26) Aureau, D., Ozanam, F., Allongue, P., and Chazalviel, J. N., The Titration of CarboxylTerminated Monolayers Revisited: In Situ Calibrated Fourier Transform Infrared Study of WellDefined Monolayers on Silicon, Langmuir 24 (17), 9440 (2008).

(27) M. L. Munford, R. C., P. Allongue, The preparation of ideally ordered flat H-Si(111) surfaces, Sensors and Materials 13 (5), 259 (2001).

(28) Faucheux, A., Yang, F., Allongue, P., De Villeneuve, C. H., Ozanam, F., and Chazalviel, J. N., Thermal decomposition of alkyl monolayers covalently grafted on (111) silicon, Appl. Phys. Lett. 88 (19) (2006); Gorostiza, P., de Villeneuve, C. H., Sun, Q. Y., Sanz, F., Wallart, X., Boukherroub, R., and Allongue, P., Water exclusion at the nanometer scale provides long-term passivation of silicon(111) grafted with alkyl monolayers, Journal of Physical Chemistry B 110 (11), 5576 (2006).

(29) García, R. and Pérez, R., Dynamic atomic force microscopy methods, Surface Science Reports 47 (6-8), 197 (2002).

(30) Garcia, R. and San Paulo, A., Amplitude curves and operating regimes in dynamic atomic force microscopy, Ultramicroscopy 82 (1-4), 79 (2000); San Paulo, A. and Garcia, R., Highresolution imaging of antibodies by tapping-mode atomic force microscopy: Attractive and repulsive tip-sample interaction regimes, Biophysical Journal 78 (3), 1599 (2000). 
(31) Gorostiza, P., Henry de Villeneuve, C., Sun, Q. Y., Sanz, F., Wallart, X., Boukherroub, R., and Allongue, P., Water Exclusion at the Nanometer Scale Provides Long-Term Passivation of Silicon (111) Grafted with Alkyl Monolayers, The Journal of Physical Chemistry B 110 (11), 5576 (2006).

(32) Arnold, R., Azzam, W., Terfort, A., and Wöll, C., Preparation, Modification, and Crystallinity of Aliphatic and Aromatic Carboxylic Acid Terminated Self-Assembled Monolayers, Langmuir 18 (10), 3980 (2002).

(33) Levy, O., Markovits, G. Y., and Perry, I., Thermodynamics of aggregation of long chain carboxylic acids in benzene, The Journal of Physical Chemistry 79 (3), 239 (1975).

(34) Ruiz, F.-J., Rubio, S., and Pérez-Bendito, D., Water-Induced Coacervation of Alkyl Carboxylic Acid Reverse Micelles: Phenomenon Description and Potential for the Extraction of Organic Compounds, Analytical Chemistry 79 (19), 7473 (2007).

(35) Correa, N. M., Silber, J. J., Riter, R. E., and Levinger, N. E., Nonaqueous Polar Solvents in Reverse Micelle Systems, Chemical Reviews 112 (8), 4569 (2012).

(36) Rossignol, S., Tinel, L., Bianco, A., Passananti, M., Brigante, M., Donaldson, D. J., and George, C., Atmospheric photochemistry at a fatty acid-coated air-water interface, Science $\mathbf{3 5 3}$ (6300), 699 (2016). 
Figures:

$\Gamma_{\mathrm{SOL}}=0.2$
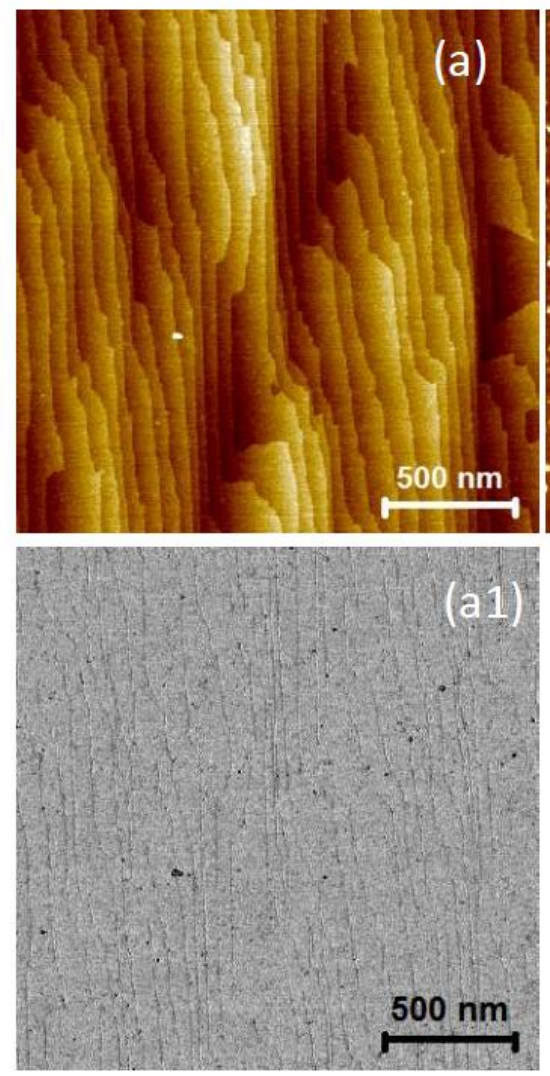

$10^{-4}$

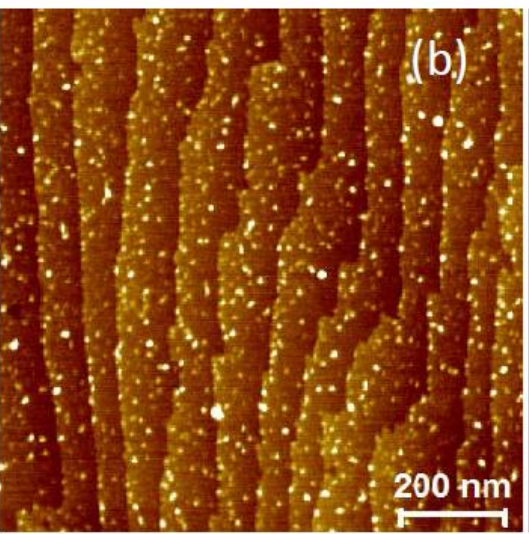

(b1)

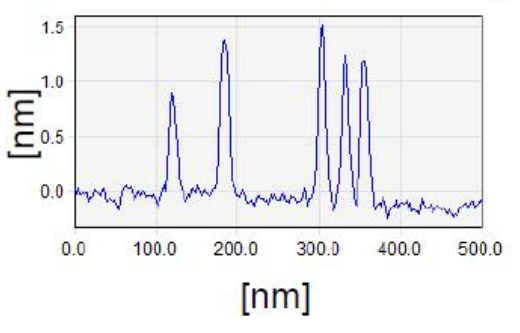

$10^{-4}$
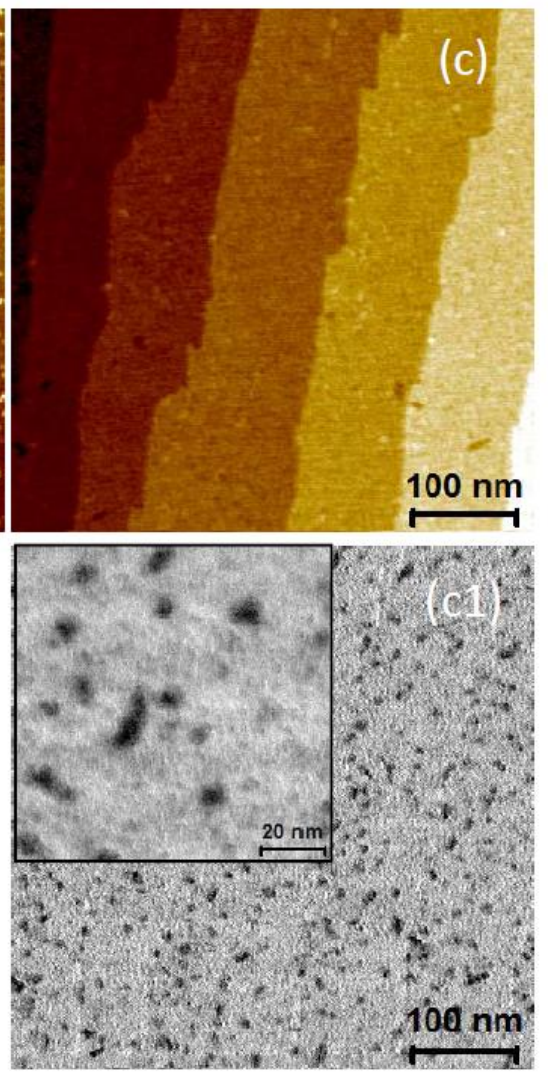

Figure 1: AFM topography and phase images of mixed monolayers grafted on $\mathrm{Si}(111)$ from solutions with a high acid concentration (acid-molecule fraction $\Gamma_{\mathrm{SOL}}$ of 0.2 ) or at extreme dilution $\left(\Gamma_{\mathrm{SOL}}=10^{-4}\right) . \Gamma_{\mathrm{SOL}}$ is indicated above the topographic images. Samples (a) and (b) were rinsed in hot solvents (see text). Image (c) corresponds to the sample shown in (b) but after mechanical cleaning. A cross-section of image (b) is shown in (b1). The inset in image (c) is an image of the same sample at a larger magnification. 


$$
\Gamma_{\mathrm{SOL}}=0.1
$$
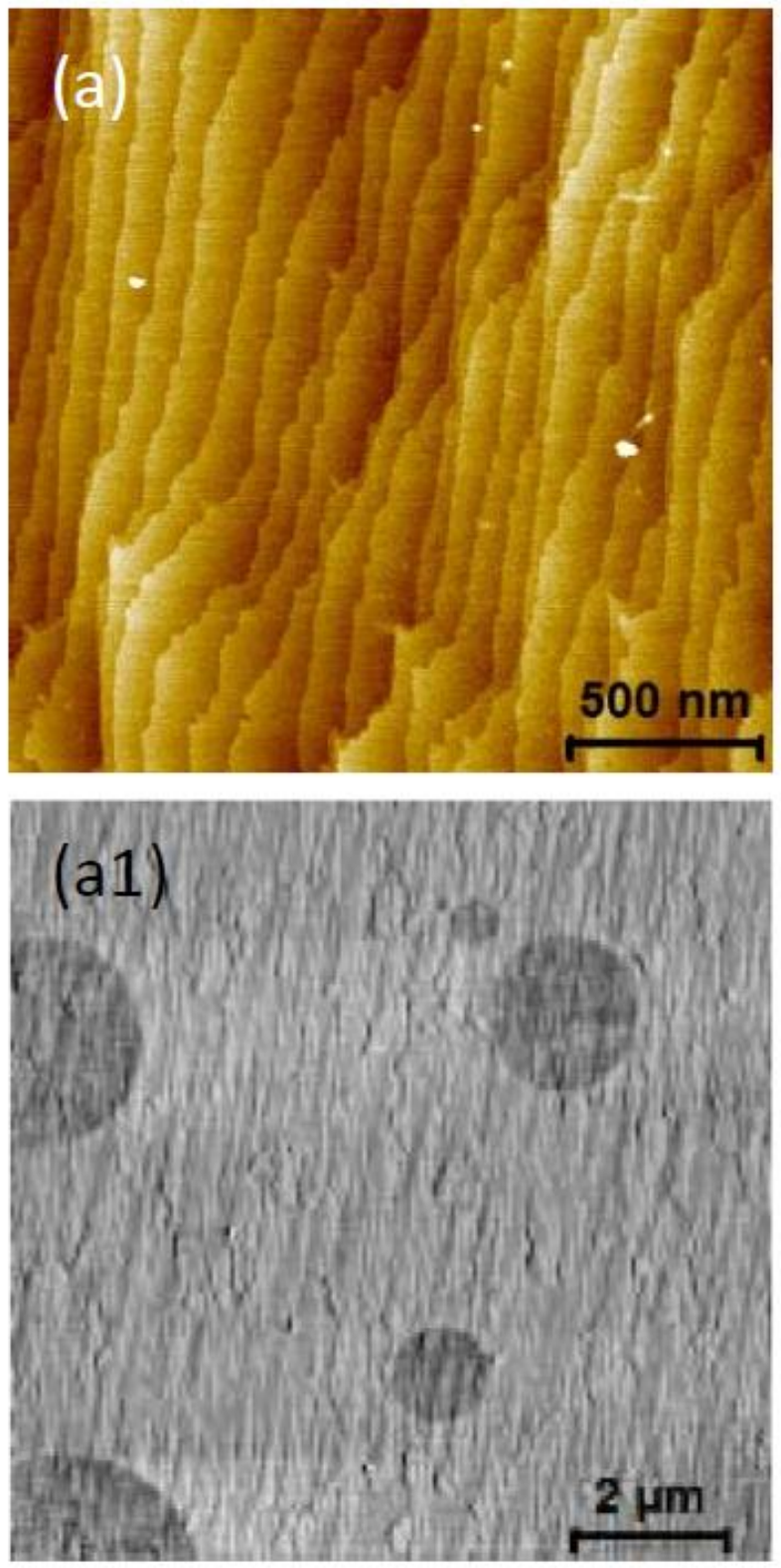

0.01
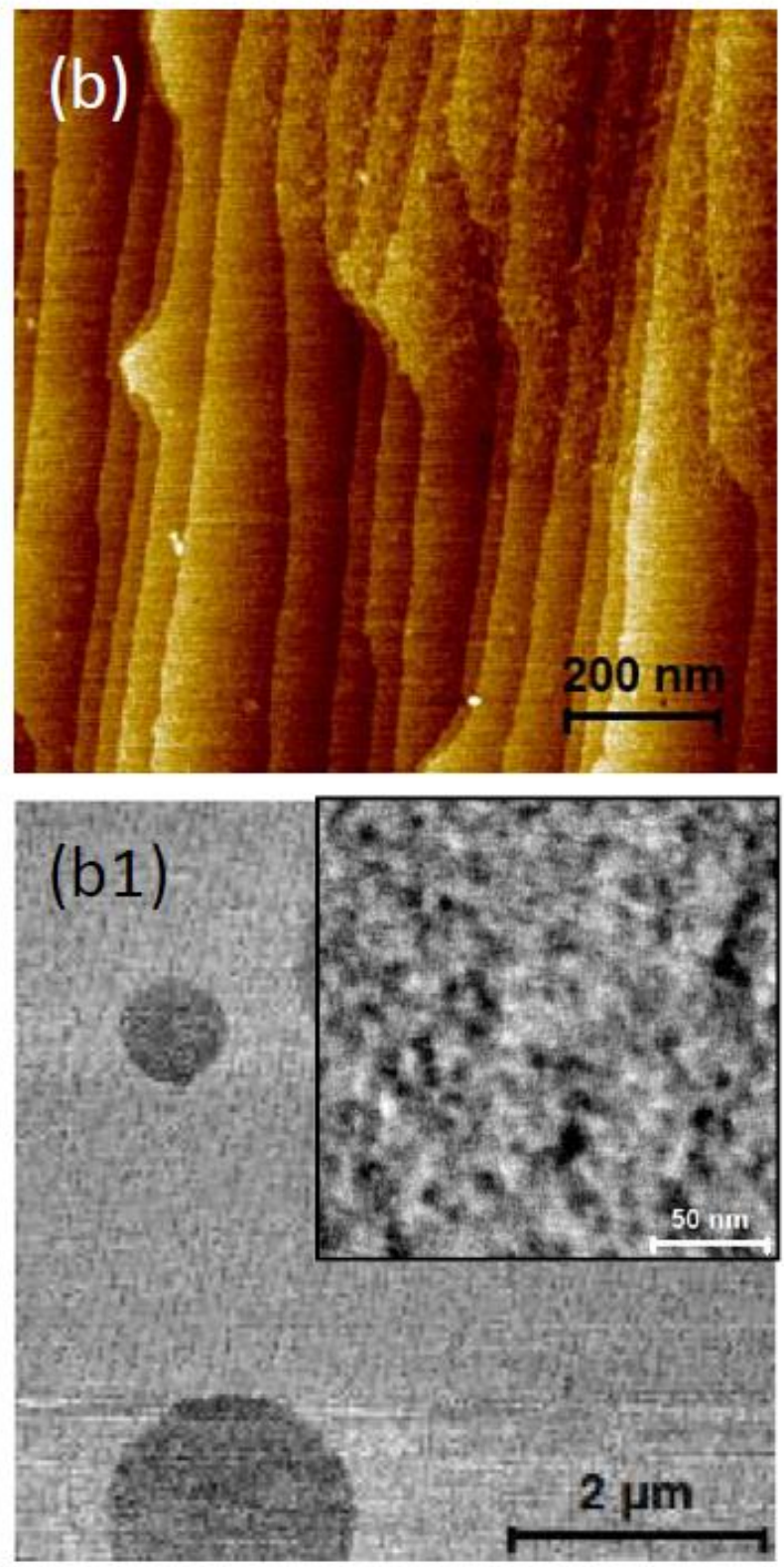

Figure 2: AFM topography and phase images of mixed monolayers grafted on $\mathrm{Si}(111)$ from dilute acid solutions $\Gamma_{\mathrm{SOL}}$ of 0.1 and 0.01 , as indicated above the topographic images). Samples were rinsed in hot solvents and a mechanical cleaning was applied to remove a large excess of matter left after grafting (see text). The inset in image (b) is an image of the same sample at a larger magnification outside the dark domains. The surfaces are imaged after specific rinsing and cleaning (see text). Complementary observations are given in Supplementary Information (Fig. S1, S4). 


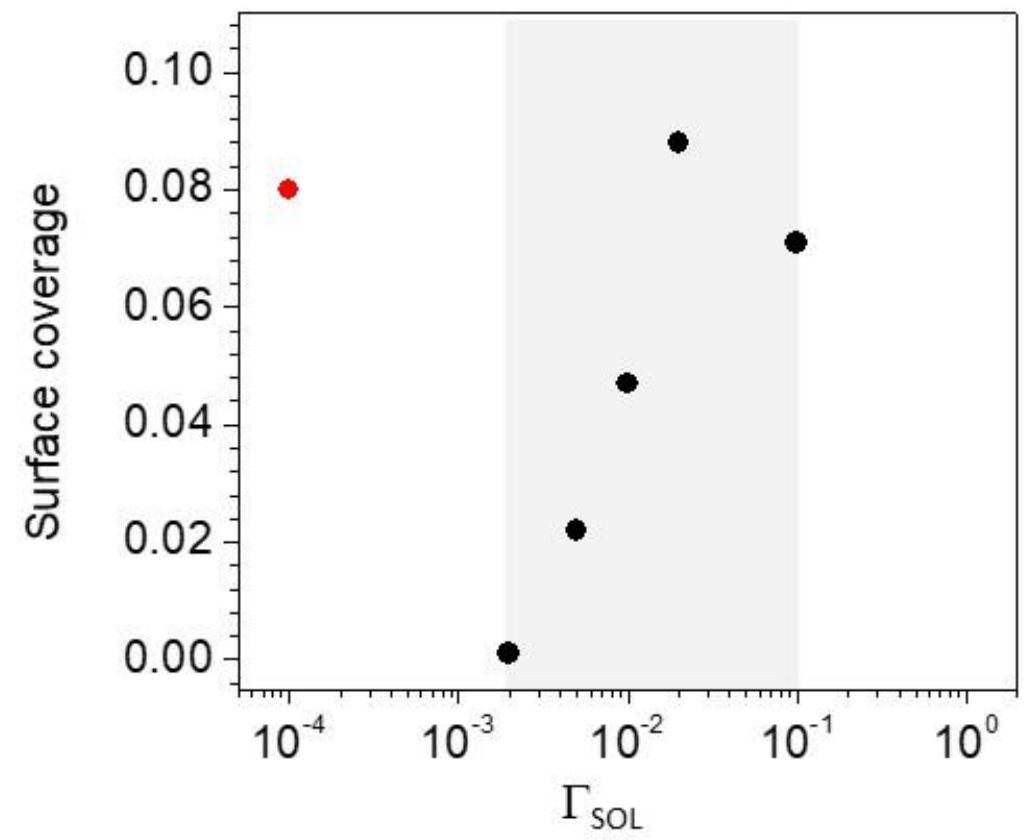

Figure 3: Surface coverage of the dark domains observed on the phase images (Figs. 1 and 2) as a function of the acid-chain fraction in solution $\Gamma_{\mathrm{SOL}}$. The gray shaded region corresponds to $\mu-$ domains and the red data point corresponds to the dark domains at extreme dilution (see Fig. 1c1). No domain is observed above 0.1 (Fig. 1a1). 

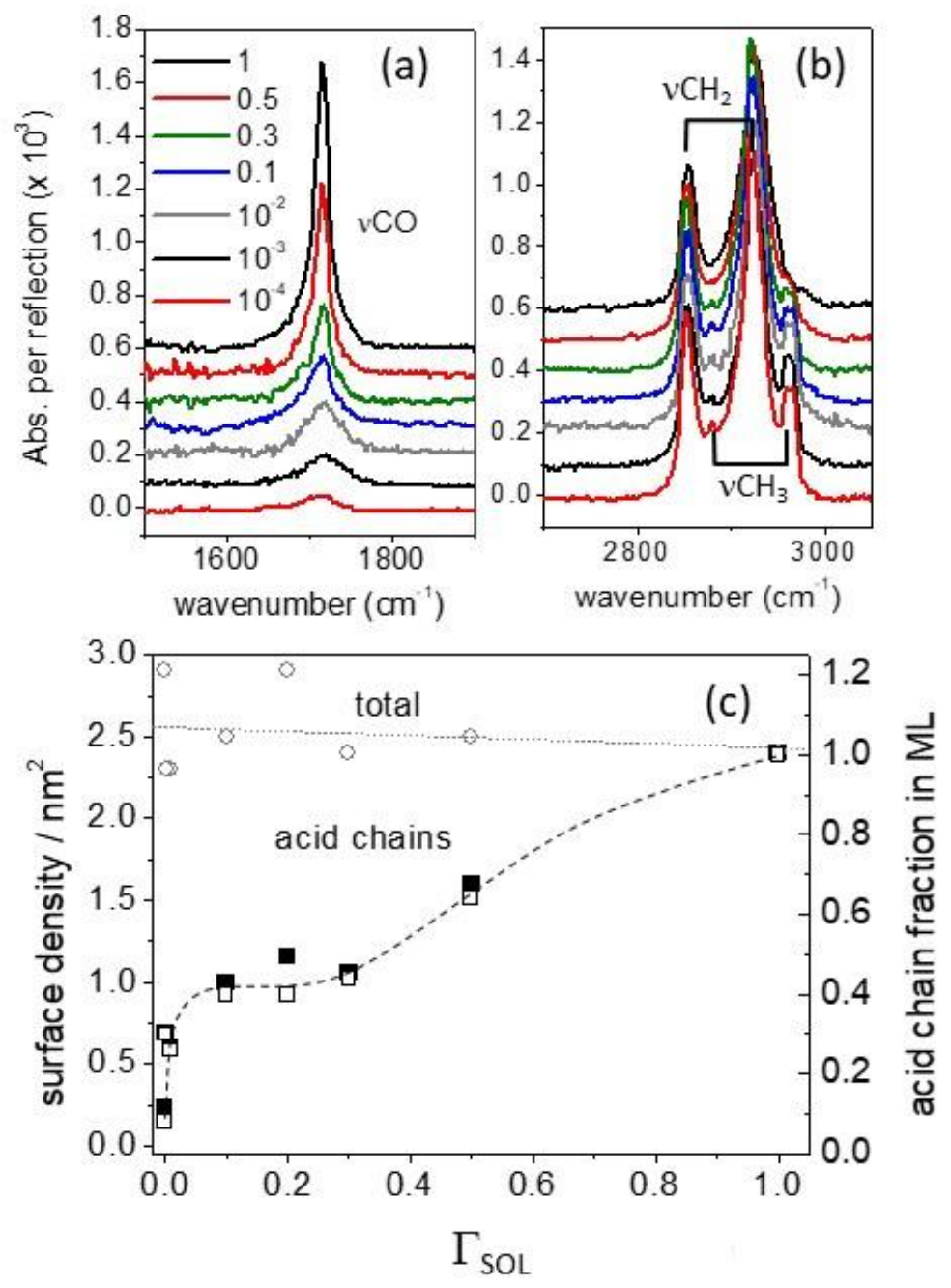

Figure 4: FTIR characterizations of cleaned mixed monolayers grafted in solutions containing a variable content of acid molecules. (a-b) Narrow FTIR spectra in the $v$ CO range $\left(1600-1800 \mathrm{~cm}^{-}\right.$ ${ }^{1}$ ) and $\mathrm{vCH}$ range $\left(2800-3000 \mathrm{~cm}^{-1}\right): \Gamma_{\mathrm{SOL}}=10^{-4}$ to 1 from bottom to top. (c) Surface density (left y-axis) of acid-chains (full squares) and total chains (acid+decyl) (open circles) as a function of the acid-molecule fraction $\Gamma_{\mathrm{SOL}}$ in solution. The dashed line is a guide to eye. The acid-chain fraction in the monolayer $\Gamma_{\mathrm{SURF}}$ is shown as open squares (right y-axis). 


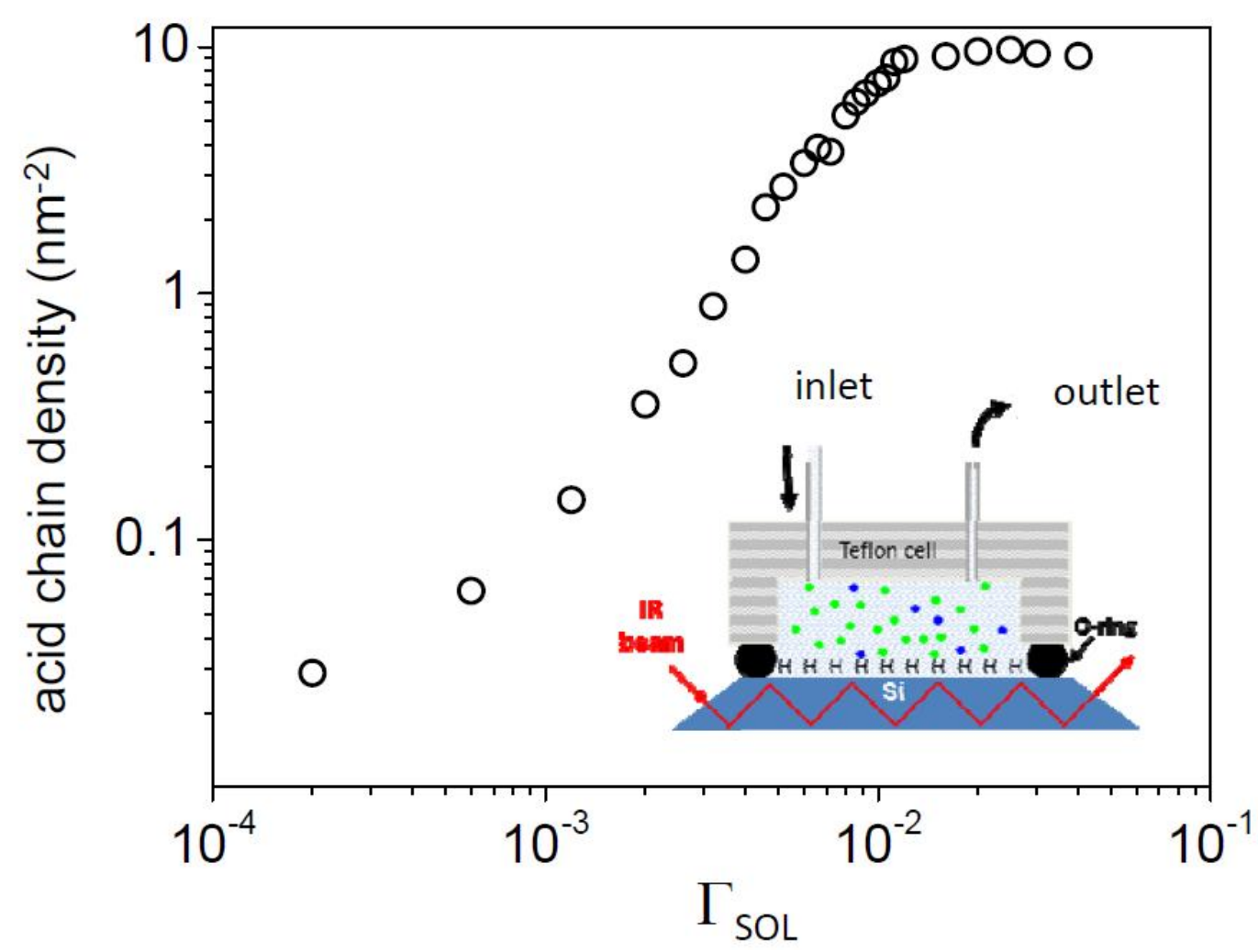

Figure 5: Adsorption experiment on $\mathrm{H}-\mathrm{Si}(111)$ exposed to a mixed solution in the dark, i.e., no grafting is occurring. Surface density of acid chains is plotted as a function of the acid-molecule fraction in solution $\Gamma_{\text {SOL }}$. The log-log scale highlights that adsorption begins at very low $\Gamma_{\text {SOL }}$. The data points are derived from in-situ FTIR measurement using a custom cell sketched in the inset. See text and supplementary information, Fig. S6, for details. 

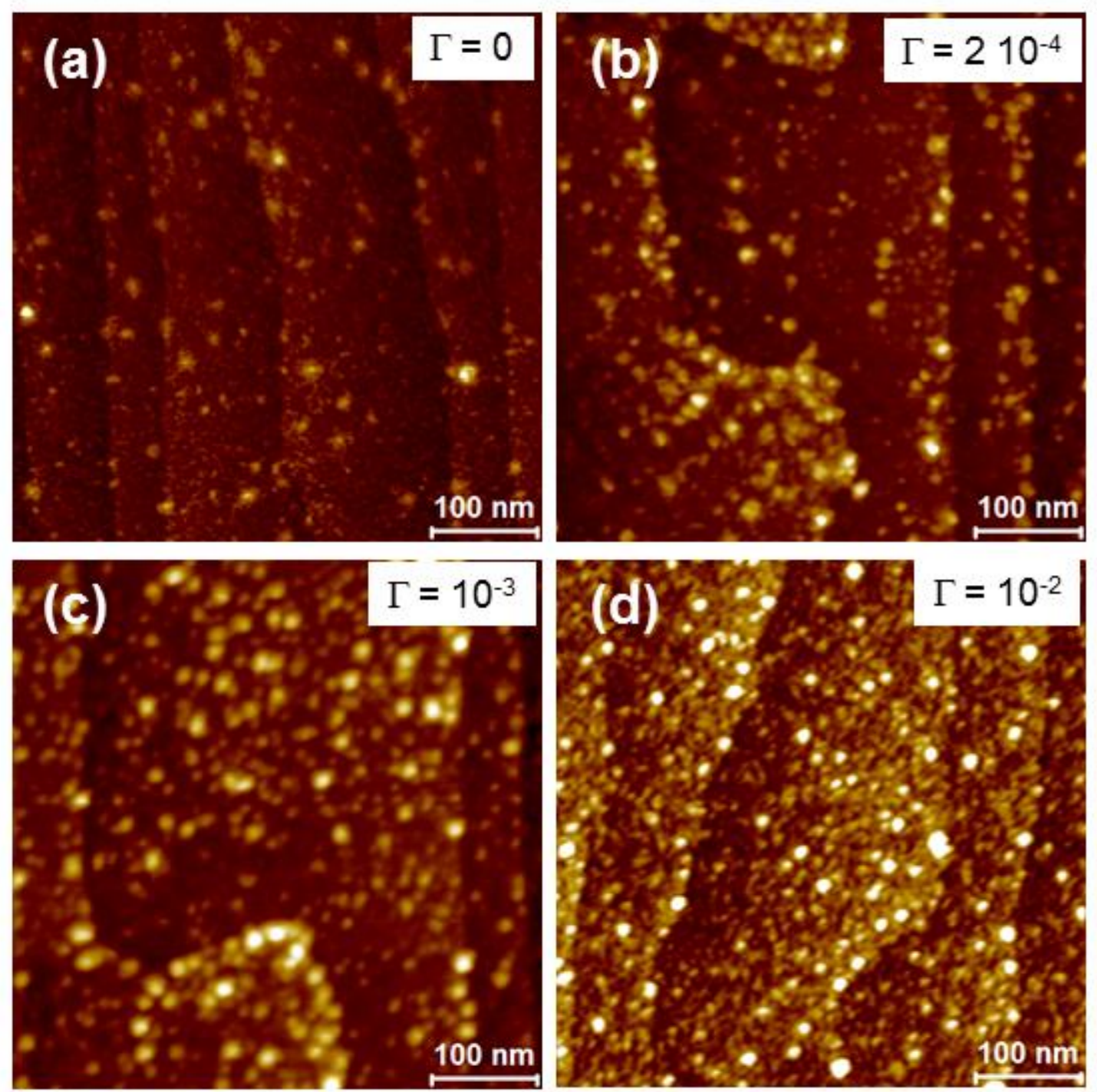

Figure 6: AFM images of H-terminated $\mathrm{Si}(111)$ surfaces exposed in the dark and at room temperature to grafting solutions with variable acid fractions $\Gamma_{\mathrm{SOL}}(\Delta \mathrm{Z}$ range $=3 \mathrm{~nm}$ for all images). All spots in (b-d) are $\sim 1.5 \mathrm{~nm}$ mean high while they are only $0.4 \mathrm{~nm}$ high in (a). See text. Each surface was exposed for 20 min to the grafting solution and rinsed in boiling dichloromethane for $5 \mathrm{~min}$. 


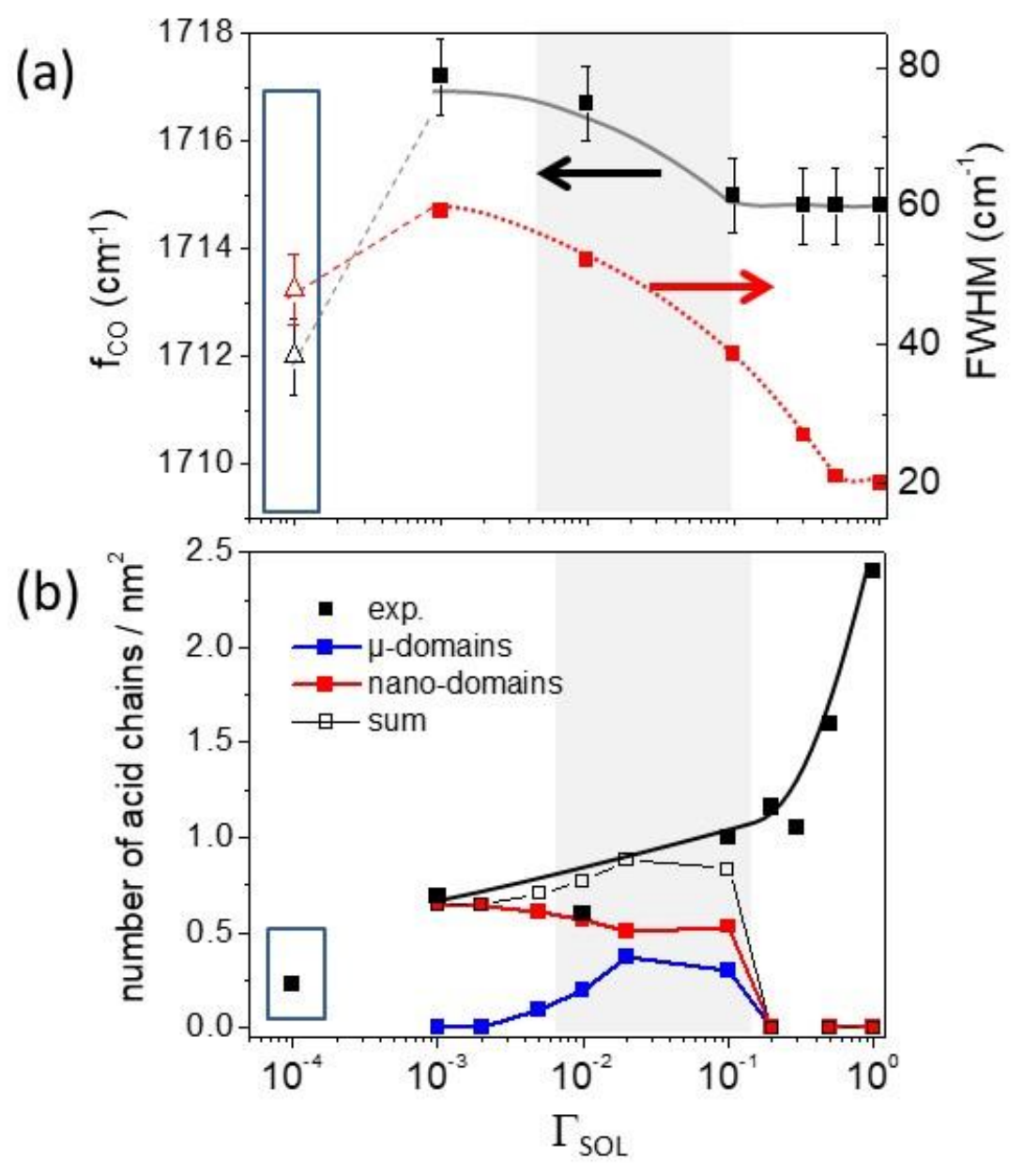

Figure 7: (a) Variations of the frequency ( $\left.\mathrm{f}_{\mathrm{CO}}\right)$ and width (fwhm) of the $v \mathrm{CO}$ IR band as a function of the acid-molecule fraction in solution $\Gamma_{\mathrm{SOL}} . \mathrm{f}_{\mathrm{CO}}$ and fwhm are measured in the spectra shown in Fig. 4a. (b) Experimental density of acid chains (black squares) as a function of $\Gamma_{\mathrm{SOL}}$ and plots of the normalized numbers of acid chains in $\mu$-domains $N_{\mu}$ (blue) and in n-domains $N_{\mathrm{n}}$ (red) and sum (open squares). These plots have been calculated, with $\theta_{\mathrm{n}}=0.35, D_{\mu}=1.7 / \mathrm{nm}^{2}$ and $D_{\mathrm{n}}=1.8 / \mathrm{nm}^{2}$ (hypothesis of full segregation condition : $N_{\mu}+N_{\mathrm{n}} \sim N_{\text {acid }}$ ). See text for details. The shaded area underlines the region where the contribution of $\mu$-domains to the total acid surface density is not negligible. The symbols at $\Gamma_{\mathrm{SOL}}=10^{-4}$ correspond to the dark domains observed in Fig. 1c1. 
(a)

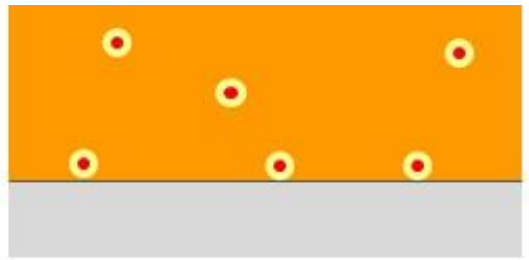

(b)

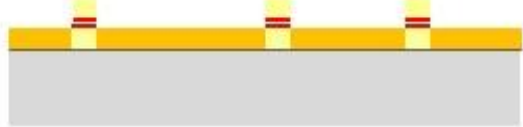

(c)
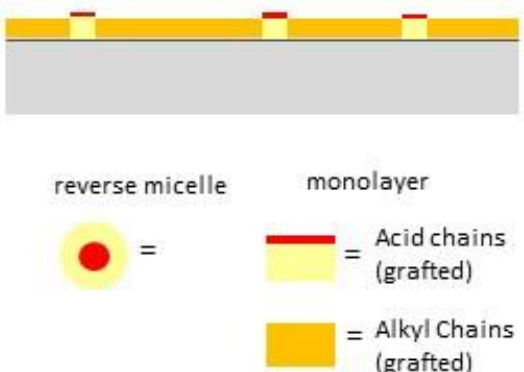

Figure 8: Schematic grafting mechanism at extreme dilution of the mixed solution $\left(\Gamma_{\mathrm{SOL}}=10^{-4}\right)$. (a) Reverse micelles formed in solution are adsorbing on the H-terminated surface in the dark. (b) Upon UV illumination grafting occurs and the resulting monolayer consists of an alkyl monolayer with isolated bilayer islands composed of acid chains. (c) The dimers are disrupted by additional mechanical cleaning, which leaves an atomically smooth surface. This mechanism explains the observations in Figs. 1b-1c-. 
TOC:
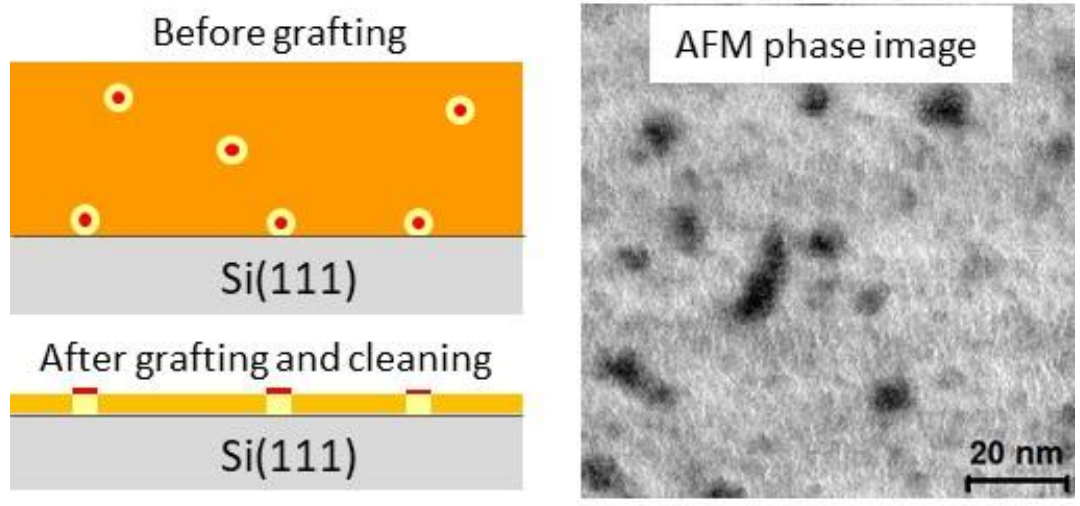

After grafting and cleaning

Si(111) 